PROCEEDINGS OF THE

AMERICAN MATHEMATICAL SOCIETY

Volume 137, Number 12, December 2009, Pages 4049-4063

S 0002-9939(09)10075-8

Article electronically published on August 7, 2009

\title{
EXPLICIT UPPER BOUNDS FOR $L$-FUNCTIONS ON THE CRITICAL LINE
}

\author{
VORRAPAN CHANDEE
}

(Communicated by Ken Ono)

\begin{abstract}
We find an explicit upper bound for general $L$-functions on the critical line, assuming the Generalized Riemann Hypothesis, and give as illustrative examples its application to some families of $L$-functions and Dedekind zeta functions. Further, this upper bound is used to obtain lower bounds beyond which all eligible integers are represented by Ramanujan's ternary form and Kaplansky's ternary forms. This improves on previous work by Ono and Soundararajan on Ramanujan's form and by Reinke on Kaplansky's forms with a substantially easier proof.
\end{abstract}

\section{INTRODUCTION}

Finding upper bounds for $L$-functions on the critical line is an interesting problem in analytic number theory. One classical bound is the convexity bound, which follows from the Phragmen-Lindelöf principle and the approximate functional equation of the $L$-function. For any $\epsilon>0$, the convexity bound is

$$
L(f, s) \ll q(f, s)^{1 / 4+\epsilon},
$$

where $\Re s=\frac{1}{2}$ and $q(f, s)$ is the analytic conductor of the $L$-function, whose definition we recall later. However, for many applications this convexity bound is not sufficient, and one needs to improve it for relevant $L$-functions by reducing the exponent $1 / 4$ by a positive number. This is known as the subconvexity problem. Although such subconvexity bounds have been derived in many cases, we are still far from proving the conjectured order, even for the Riemann zeta function. The Lindelöf hypothesis states that for any $\epsilon>0$,

$$
L(f, s) \ll q(f, s)^{\epsilon},
$$

where $\Re s=\frac{1}{2}$, and the implied constant depends on $\epsilon$. This is a well-known consequence of the Generalized Riemann Hypothesis (GRH) (see e.g. Corollary 5.20, p. 116, in [5]). Indeed, in the process of deducing the Lindelöf hypothesis from the GRH, we obtain that for some $A>0$

$$
L\left(f, \frac{1}{2}\right) \ll \exp \left(A \frac{\log q\left(f, \frac{1}{2}\right)}{\log \log q\left(f, \frac{1}{2}\right)}\right) .
$$

Received by the editors April 15, 2009, and, in revised form, April 20, 2009.

2000 Mathematics Subject Classification. Primary 11M41; Secondary 11E25.

Key words and phrases. L-functions, critical line, ternary quadratic form.

(C)2009 American Mathematical Society Reverts to public domain 28 years from publication 
In this paper, instead of giving asymptotic upper bounds for $L$-functions, we will compute an explicit upper bound for $L$-functions on the critical line, assuming GRH. This type of upper bound is useful for certain applications. For example, Ono and Soundararajan 7 used explicit upper bounds (on GRH) for $L$-functions at the critical point to show that all odd numbers larger than $2 \cdot 10^{10}$ are represented by Ramanujan's form $x^{2}+y^{2}+10 z^{2}$. Using our work here, we can give a substantially easier proof of their results. Moreover our bounds show that all odd numbers larger than $3 \cdot 10^{7}$ are represented, so that much less computation is required. We anticipate that these results would be useful in studying representation questions for general ternary quadratic froms. We will give more details on this problem, together with other examples, in $\S 4$.

To calculate the explicit upper bound, we will follow Soundararajan's technique from [11. The main idea of the technique is to write $\log \left|L\left(f, \frac{1}{2}\right)\right|$ as a short sum over prime powers and a sum over nontrivial zeros of $L(f, s)$. Furthermore in finding our upper bound for $\log \left|L\left(f, \frac{1}{2}\right)\right|$, the contribution of nontrivial zeros can be ignored. The short sum over prime powers can be explicitly bounded both numerically and in terms of analytic conductor, which will be defined below. We will give details of the proof in $\S 2$. Before we state the main theorem and corollaries, here is some basic notation that we will use throughout the paper.

From [5, p. 94], $L(f, s)$ is an $L$-function if it satisfies the following properties.

I. It is a Dirichlet series with Euler product of degree $d \geq 1$,

$$
L(f, s)=\sum_{n \geq 1} \lambda_{f}(n) n^{-s}=\prod_{p}\left(1-\alpha_{1}(p) p^{-s}\right)^{-1} \cdots\left(1-\alpha_{d}(p) p^{-s}\right)^{-1}
$$

and

$$
-\frac{L^{\prime}}{L}(f, s)=\sum_{\substack{n=p^{l} \\ l \geq 1}} \frac{\left(\alpha_{1}^{l}(p)+\cdots+\alpha_{d}^{l}(p)\right) \log p}{n^{s}}=\sum_{n} \frac{a(n)}{n^{s}}
$$

with $\lambda_{f}(1)=1,\left|\alpha_{p}\right|<p$ for all $p$. The series and Euler products are absolutely convergent for $\Re(s)>1$.

II. Let

$$
\gamma(f, s)=\pi^{-d s / 2} \prod_{j=1}^{d} \Gamma\left(\frac{s+k_{j}}{2}\right)
$$

be a gamma factor. Since we will assume GRH, $\Re k_{j} \geq 0$. This condition tells us that $\gamma(f, s)$ has no zero in $\mathbb{C}$ and no pole for $\Re(s) \geq 0$. Let an integer $q(f) \geq 1$ be a conductor of $L(f, s)$ such that $\alpha_{i}(p) \neq 0$ for $p$ not dividing $q(f)$. Let

$$
\Lambda(f, s)=q(f)^{s / 2} \gamma(f, s) L(f, s) .
$$

$L(f, s)$ must satisfy the functional equation

$$
\Lambda(f, s)=\epsilon(f) \bar{\Lambda}(f, 1-s),
$$

where $|\epsilon(f)|=1$, and $\bar{\Lambda}(f, s)=\overline{\Lambda(f, \bar{s})}$. We define the analytic conductor of $L(s, f)$ to be

$$
C=\frac{q(f)}{\pi^{d}} \prod_{j=1}^{d}\left|\frac{1}{4}+\frac{k_{j}}{2}\right| .
$$


In this paper, we will assume that $\Lambda(f, s)$ has no zero or pole at $\Re s=1$. Note that by the functional equation, this implies the same holds at $\Re s=0$.

Our main theorem will be an upper bound for $\log L\left(f, \frac{1}{2}\right)$ in terms of the sum over prime powers and the analytic conductor (Theorem 2.1 in $\S 2$ ). As a corollary, we derive the Lindelöf hypothesis from the main theorem. In fact, we can find an explicit constant $A$ in (1) as below.

Corollary 1.1. Let $L(f, s)$ be an L-function satisfying the conditions above, and let $C$ be defined as in (6). Furthermore, assume that $L(f, s)$ satisfies Ramanujan's conjecture. Then for $\log \log C \geq 10$,

$$
\log \left|L\left(f, \frac{1}{2}\right)\right| \leq \frac{23 d}{25} \frac{\log C}{\log ^{2} \log C}+\frac{3}{8} \frac{\log C}{\log \log C} .
$$

Remark 1. Without Ramanujan's conjecture, we instead obtain

$$
\log \left|L\left(f, \frac{1}{2}\right)\right| \leq \frac{9}{8} \frac{\log C}{\log \log C}+O\left(\frac{d \log C}{\log ^{2} \log C}\right) .
$$

Remark 2. It may be possible to improve the explicit constant $3 / 8$ appearing in Corollary 1.1 by using a different kernel in the proof of Lemma 2.4.

Applying Corollary 1.1 to families of $L$-functions that satisfy Ramanujan's conjecture, we easily obtain the explicit upper bound for these $L$-functions in terms of their analytic conductor. We will illustrate our results using three examples of families of $L$-functions: Dirichlet $L$-functions, holomorphic cusp forms, and the Riemann zeta function with varying height on the critical line.

Corollary 1.2. Let $\chi$ be a primitive even Dirichlet character modulo q. Let $\tilde{C}=$ $q\left(|t|+\frac{1}{2}\right)$. If $\log \log \tilde{C} \geq 10$, then for any $t$,

$$
\left|L\left(\frac{1}{2}+i t, \chi\right)\right| \leq \exp \left(\frac{23}{25} \frac{\log \tilde{C}}{\log ^{2} \log \tilde{C}}+\frac{3}{8} \frac{\log \tilde{C}}{\log \log \tilde{C}}\right) .
$$

Corollary 1.3. Let $f(s)=\sum_{n \geq 1} \lambda(n) q^{n}$ be a holomorphic cusp form of weight $k \geq 1$ and level $q$, and $L(f, s)=\sum_{n \geq 1} \frac{\lambda(n)}{n^{s}}$ be an L-function associated to $f$. Define $\tilde{C}=q k^{2}$. If $\log \log \tilde{C} \geq 10$, then

$$
\left|L\left(f, \frac{1}{2}\right)\right| \leq \exp \left(\frac{46}{25} \frac{\log \tilde{C}}{\log ^{2} \log \tilde{C}}+\frac{3}{8} \frac{\log \tilde{C}}{\log \log \tilde{C}}\right) .
$$

Corollary 1.4. Assume $T \leq t \leq 2 T$. Let $\tilde{C}=T$. Also assume that $\log \log \tilde{C} \geq 10$. Then

$$
\left|\zeta\left(\frac{1}{2}+i t\right)\right| \leq \exp \left(\frac{23}{25} \frac{\log \tilde{C}}{\log ^{2} \log \tilde{C}}+\frac{3}{8} \frac{\log \tilde{C}}{\log \log \tilde{C}}\right) .
$$

Remark 3 . The analytic conductor is a measure of the complexity of the $L$-function. For our examples above, $C \approx \tilde{C}$. Hence we can apply Corollary[1.1 to $\tilde{C}$. The details will be discussed in $\S 2$.

Finally, with some modifications, we can apply Theorem 2.1 to Dedekind zeta functions. We will prove the following corollary in $\S 3$. 
Corollary 1.5. Let $K / \mathbb{Q}$ be a number field of degree $d$. The Dedekind zeta function

$$
\zeta_{K}(s)=\prod_{\mathfrak{p}}\left(1-(N \mathfrak{p})^{-s}\right)^{-1}
$$

is an L-function of degree $d$ with conductor $q$ equal to the absolute value of the discriminant of $K$. If $\log \log C \geq 10$, then

$$
\left|\zeta_{K}\left(\frac{1}{2}\right)\right| \leq 2.33 \exp \left(\frac{23 d}{25} \frac{\log C}{\log ^{2} \log C}+\frac{3}{8} \frac{\log C}{\log \log C}\right) .
$$

\section{Proof of the main theorem and its Corollaries}

We will now prove an explicit upper bound for $L\left(f, \frac{1}{2}\right)$ in terms of a sum over powers of primes and parameters associated with $L(f, s)$. Then we will simplify the sum and those constants to derive the corollaries stated in the introduction. Our main theorem is

Theorem 2.1. Assume the $G R H$ and $\Lambda(f, s)$ has no pole or zero at $s=0,1$. Let $\lambda_{0}=0.4912 \cdots$ denote the unique positive real number satisfying $e^{-\lambda_{0}}=\lambda_{0}+\lambda_{0}^{2} / 2$. Then for all $\frac{\log x}{2} \geq \lambda \geq \lambda_{0}$ and $\log x \geq 2$, we have

$$
\begin{aligned}
\left|L\left(f, \frac{1}{2}\right)\right| \leq & \Re \sum_{n \leq x} \frac{a(n)}{n^{\frac{1}{2}+\frac{\lambda}{\log x}} \log n} \frac{\log \frac{x}{n}}{\log x} \\
& +\left(\frac{1+\lambda}{2}\right) \frac{\log C}{\log x}+\frac{\left(\lambda^{2}+\lambda\right) d}{\log ^{2} x}+\frac{4 d e^{-\lambda}}{x^{1 / 2} \log ^{2} x} .
\end{aligned}
$$

Remark 4. The above easily leads to an upper bound for $\log \left|L\left(f, \frac{1}{2}+i t\right)\right|$ as well. Indeed, we can set $L_{\text {new }}(f, s)=L(f, s+i t)$ so that $L\left(f, \frac{1}{2}+i t\right)=L_{\text {new }}\left(f, \frac{1}{2}\right)$. $L_{\text {new }}(f, s)$ satisfies the functional equation (5), but with $k_{j}+i t$ in the gamma factor in (4).

Proof of Theorem 2.1, Let $\rho=\frac{1}{2}+i \gamma$ run over the nontrivial zeros of $L(f, s)$. Define

$$
G(s)=\Re \sum_{\rho} \frac{1}{s-\rho}=\sum_{\rho} \frac{\sigma-\frac{1}{2}}{\left(\sigma-\frac{1}{2}\right)^{2}+(\gamma-t)^{2}} .
$$

To prove Theorem 2.1, we need to use the Hadamard factorization formula below. The proof can be found in [5] (see Theorem 5.6).

Proposition 2.2. Let $L(f, s)$ be an $L$-function and $s=\sigma+i t$. There exist constants $a, b$ such that

$$
\Lambda(f, s)=e^{a+b s} \prod_{\rho \neq 0,1}\left(1-\frac{s}{\rho}\right) e^{s / \rho},
$$

where $\rho$ runs over all zeros of $\Lambda(f, s)$. Hence

$$
-\frac{L^{\prime}}{L}(f, s)=\frac{1}{2} \log \frac{q}{\pi^{d}}+\frac{1}{2} \sum_{j=1}^{d} \frac{\Gamma^{\prime}}{\Gamma}\left(\frac{s+k_{j}}{2}\right)-b-\sum_{\rho \neq 0,1}\left(\frac{1}{s-\rho}+\frac{1}{\rho}\right),
$$

is uniformly and absolutely convergent in compact subsets that have no zeros or poles. Furthermore, $\Re\left(-b+\sum \frac{1}{\rho}\right)=0$. 
By (7), since $\Re\left(-b+\sum \frac{1}{\rho}\right)=0$, if $L(f, s) \neq 0$, we have

$$
-\Re \frac{L^{\prime}}{L}(f, s)=\frac{1}{2} \log \frac{q}{\pi^{d}}+\frac{1}{2} \sum_{j=1}^{d} \Re \frac{\Gamma^{\prime}}{\Gamma}\left(\frac{s+k_{j}}{2}\right)-G(s) .
$$

We need the following lemma to find the upper bound for $\Re \frac{\Gamma^{\prime}}{\Gamma}\left(\frac{s+k_{j}}{2}\right)$. The proof of the lemma can be found in the Appendix (see $\S 5$ ).

Lemma 2.3. Let $z=x+i y$, where $x \geq \frac{1}{4}$. Then

$$
\Re \frac{\Gamma^{\prime}}{\Gamma}(z) \leq \log |z| \text {. }
$$

From Lemma 2.3 and (8), we obtain

$$
-\Re \frac{L^{\prime}}{L}(f, s) \leq \frac{1}{2} \log \frac{q}{\pi^{d}}+\frac{1}{2} \sum_{j=1}^{d} \log \left|\frac{s+k_{j}}{2}\right|-G(s) .
$$

When we integrate the inequality above as $\sigma$ varies from $\frac{1}{2}$ to $\sigma_{0}=\frac{1}{2}+\frac{\lambda}{\log x}$, we get

$$
\begin{aligned}
\log \left|L\left(f, \frac{1}{2}\right)\right|-\log \left|L\left(f, \sigma_{0}\right)\right| \\
\leq \frac{\lambda}{2 \log x} \log \frac{q}{\pi^{d}}+\frac{1}{2} \sum_{j=1}^{d} \int_{\frac{1}{2}}^{\sigma_{0}} \log \left|\frac{\sigma+k_{j}}{2}\right| d \sigma-\frac{1}{2} \sum_{\rho} \log \frac{\left(\sigma_{0}-\frac{1}{2}\right)^{2}+\gamma^{2}}{\gamma^{2}} \\
\quad \leq \frac{\lambda}{2 \log x} \log \frac{q}{\pi^{d}}+\left(\frac{\lambda}{2 \log x}\right) \sum_{j=1}^{d} \log \left|\frac{\sigma_{0}+k_{j}}{2}\right|-\frac{1}{2}\left(\sigma_{0}-\frac{1}{2}\right) G\left(\sigma_{0}\right) \\
\quad \leq \frac{\lambda}{2 \log x} \log C+\frac{\lambda^{2} d}{\log ^{2} x}-\frac{\lambda}{2 \log x} G\left(\sigma_{0}\right),
\end{aligned}
$$

where we use the fact that $\log \left(1+x^{2}\right) \geq \frac{x^{2}}{1+x^{2}}$, and for $\lambda \leq \frac{\log x}{2}$,

$$
\log \left|\frac{\sigma_{0}+k_{j}}{2}\right| \leq \log \left|\frac{1}{4}+\frac{k_{j}}{2}\right|+\log \left|1+\frac{\lambda}{(\log x)\left(\frac{1}{2}+k_{j}\right)}\right| \leq \log \left|\frac{1}{4}+\frac{k_{j}}{2}\right|+\frac{2 \lambda}{\log x} .
$$

To prove Theorem 2.1, we need an upper bound for $\log \left|L\left(f, \sigma_{0}\right)\right|$, which we will obtain from Lemma 2.4 below. This lemma is a version of Lemma 1 in [11 for general $L$-functions. The proof of Lemma 2.4 is essentially the same as the one in [11; however, we provide the sketch of the proof here for completeness.

Lemma 2.4. Unconditionally, for any $s$ not conciding with 1,0 or a zero of $L(f, s)$, and for any $x \geq 2$, we have

$$
\begin{aligned}
-\frac{L^{\prime}}{L}(f, s)= & \sum_{n \leq x} \frac{a(n)}{n^{s}} \frac{\log \frac{x}{n}}{\log x}+\frac{1}{\log x}\left(\frac{L^{\prime}}{L}(f, s)\right)^{\prime}+\frac{1}{\log x} \sum_{\rho \neq 0,1} \frac{x^{\rho-s}}{(\rho-s)^{2}} \\
& +\frac{1}{\log x} \sum_{j=1}^{d} \sum_{n=0}^{\infty} \frac{x^{-2 n-k_{j}-s}}{\left(2 n+k_{j}+s\right)^{2}},
\end{aligned}
$$

where $a(n)$ is defined in (3). 
Proof. With $c=\max (1,2-\sigma)$, integrating term by term using the Dirichlet series expansion of $\frac{-L^{\prime}}{L}(f, s)$, we have

$$
\frac{1}{2 \pi i} \int_{c-i \infty}^{c+i \infty}-\frac{L^{\prime}}{L}(f, s+w) \frac{x^{w}}{w^{2}} d w=\sum_{n \leq x} \frac{a(n)}{n^{s}} \log \frac{x}{n} .
$$

On the other hand, moving the line of integration to the left and calculating residues gives

$$
\begin{aligned}
\log x(- & \frac{L^{\prime}}{L}(f, s)-\frac{1}{\log x}\left(\frac{L^{\prime}}{L}(f, s)\right)^{\prime} \\
& \left.\quad-\frac{1}{\log x} \sum_{\rho \neq 0,1} \frac{x^{\rho-s}}{(\rho-s)^{2}}-\frac{1}{\log x} \sum_{j=1}^{d} \sum_{n=0}^{\infty} \frac{x^{-2 n-k_{j}-s}}{\left(2 n+k_{j}+s\right)^{2}}\right) .
\end{aligned}
$$

Equating these two expressions, we obtain the lemma.

We take $s=\sigma$ in Lemma 2.4 extract the real parts of both sides, and integrate over $\sigma$ from $\sigma_{0}$ to $\infty$. Thus for $x \geq 2$,

$$
\begin{aligned}
\log \left|L\left(f, \sigma_{0}\right)\right|=\Re( & \sum_{n \leq x} \frac{a(n)}{n^{\sigma_{0}} \log n} \frac{\log \frac{x}{n}}{\log x}-\frac{1}{\log x} \frac{L^{\prime}}{L}\left(f, \sigma_{0}\right) \\
& +\frac{1}{\log x} \sum_{\rho \neq 0,1} \int_{\sigma_{0}}^{\infty} \frac{x^{\rho-\sigma}}{(\rho-\sigma)^{2}} d \sigma \\
& \left.+\frac{1}{\log x} \int_{\sigma_{0}}^{\infty} \sum_{j=1}^{d} \sum_{n=0}^{\infty} \frac{x^{-2 n-k_{j}-\sigma}}{\left(2 n+k_{j}+\sigma\right)^{2}} d \sigma\right) .
\end{aligned}
$$

Moreover

$$
\sum_{\rho}\left|\int_{\sigma_{0}}^{\infty} \frac{x^{\rho-\sigma}}{(\rho-\sigma)^{2}} d \sigma\right| \leq \sum_{\rho} \int_{\sigma_{0}}^{\infty} \frac{x^{\frac{1}{2}-\sigma}}{\left|\rho-\sigma_{0}\right|^{2}} d \sigma=\sum_{\rho} \frac{x^{\frac{1}{2}-\sigma_{0}}}{\left|\rho-\sigma_{0}\right|^{2} \log x}=\frac{e^{-\lambda} G\left(\sigma_{0}\right)}{\lambda}
$$

and for $\Re k_{j} \geq 0$,

$$
\Re \int_{\sigma_{0}}^{\infty} \sum_{j=1}^{d} \sum_{n=0}^{\infty} \frac{x^{-2 n-k_{j}-\sigma}}{\left(2 n+k_{j}+\sigma\right)^{2}} d \sigma \leq \sum_{j=1}^{d} \frac{x^{-\Re k_{j}-\sigma_{0}}}{\log x\left|k_{j}+\sigma_{0}\right|^{2}} \sum_{n=0}^{\infty} x^{-2 n} \leq \frac{4 d e^{-\lambda}}{x^{1 / 2} \log x} .
$$

Hence using the previous two inequalities and (9), we deduce that

$$
\begin{aligned}
& \log \left|L\left(f, \sigma_{0}\right)\right| \\
\leq & \Re \sum_{n \leq x} \frac{a(n)}{n^{\sigma_{0}} \log n} \frac{\log \frac{x}{n}}{\log x}+\frac{1}{\log x}\left(\frac{1}{2} \log \frac{q}{\pi^{d}}+\frac{1}{2} \sum_{j=1}^{d} \log \left|\frac{\sigma_{0}+k_{j}}{2}\right|-G\left(\sigma_{0}\right)\right) \\
& +\frac{4 d e^{-\lambda}}{x^{1 / 2} \log ^{2} x}+\frac{e^{-\lambda} G\left(\sigma_{0}\right)}{\lambda \log x} .
\end{aligned}
$$


Adding the inequalities (10) and (12) and using (11), we get

$$
\begin{aligned}
\log \left|L\left(f, \frac{1}{2}\right)\right| \leq & \Re \sum_{n \leq x} \frac{a(n)}{n^{\sigma_{0}} \log n} \frac{\log \frac{x}{n}}{\log x}+\left(\frac{\lambda+1}{2 \log x}\right) \log C+\frac{\left(\lambda^{2}+\lambda\right) d}{\log ^{2} x} \\
& +\frac{4 d e^{-\lambda}}{x^{1 / 2} \log ^{2} x}+\frac{G\left(\sigma_{0}\right)}{\log x}\left(\frac{e^{-\lambda}}{\lambda}-1-\frac{\lambda}{2}\right) .
\end{aligned}
$$

For $\lambda \geq \lambda_{0}$, the term involving $G\left(\sigma_{0}\right)$ above gives a negative contribution, and we can omit it. Hence the theorem is proved.

Proof of Corollary 1.1. Since our $L$-function $L(f, s)$ satisfies Ramanujan's conjecture, $|a(n)| \leq d \Lambda(n)$. From Theorem 2.1 picking $\lambda=0.5$, we have

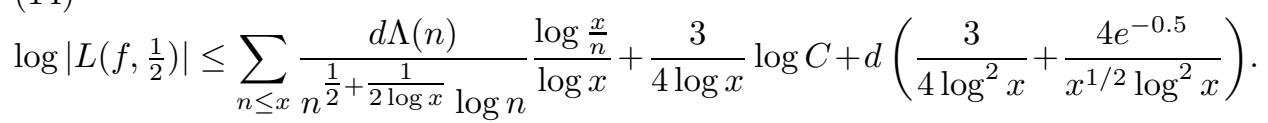

When $\log x \geq 20, \frac{3}{4 \log ^{2} x}+\frac{4 e^{-0.5}}{x^{1 / 2} \log ^{2} x} \leq 0.0019$. Now we need to find the upper bound of the sum over powers of primes. We shall prove the following inequality:

$$
\sum_{n \leq x} \frac{\Lambda(n)}{n^{\frac{1}{2}+\frac{\lambda}{\log x} \log n}} \frac{\log \frac{x}{n}}{\log x} \leq 3.675 \frac{x^{1 / 2}}{\log ^{2} x} .
$$

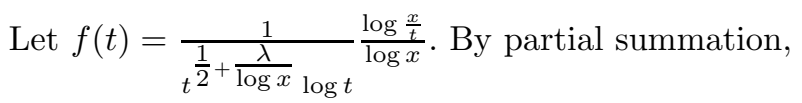

$$
\sum_{n \leq x} \frac{\Lambda(n)}{n^{\frac{1}{2}+\frac{\lambda}{\log x}} \log n} \frac{\log \frac{x}{n}}{\log x}=-\int_{2}^{x}\left(\sum_{n \leq t} \Lambda(n)\right) f^{\prime}(t) d t .
$$

Since we assume the GRH, using the result of Lemma 8 and (3.21) in [9] (pick $\delta=\frac{2}{\sqrt{2+\sqrt{x}}}$, we obtain that for $t>10$,

$$
\sum_{n \leq t} \Lambda(n) \leq t+0.0463\left(\frac{2 \sqrt{2+\sqrt{t}}+2}{\sqrt{t}}\right) t .
$$

For $t \geq 10^{5}$, we have

$$
\sum_{n \leq t} \Lambda(n)<(1.006) t
$$

The inequality is also true for $10^{5}>t \geq 2$ by numerical experiment. Therefore,

$$
\begin{aligned}
\sum_{n \leq x} \frac{\Lambda(n)}{n^{\frac{1}{2}+\frac{\lambda}{\log x} \log n} \frac{\log \frac{x}{n}}{\log x}} & \leq-1.006 \int_{2}^{x} t f^{\prime}(t) d t \\
& =1.006\left(\frac{2 \log \frac{x}{2}}{2^{1 / 2+1 /(2 \log x)} \log 2 \log x}+\int_{2}^{x} f(t) d t\right) .
\end{aligned}
$$

We change variable $t=x / y$ and obtain

$$
\int_{2}^{x} f(t) d t=\frac{e^{-\lambda} x^{1 / 2}}{\log ^{2} x} \int_{1}^{x / 2} \frac{\log y}{y^{3 / 2-1 /(2 \log x)}\left(1-\frac{\log y}{\log x}\right)} d y \leq 5.961 \frac{e^{-\lambda} x^{1 / 2}}{\log ^{2} x}
$$

where the last inequality follows because the second integral above is a decreasing function of $x$ when $x>10^{4}$. The constant appearing on the right-hand side is 
derived by substituing $x=e^{20}$ in the integral. Finally, by absorbing the constant term into the $\frac{x^{1 / 2}}{\log ^{2} x}$ term, we derive (15). Choosing $x=\log ^{2} C$ in (14) and applying (15), we prove the corollary.

Proof of Corollary 1.2, The Dirichlet $L$-function $L(s+i t, \chi)$ is an $L$-function of degree 1 with conductor $q$, and it satisfies Ramanujan's conjecture. The gamma factor is $\gamma(s)=\pi^{-s / 2} \Gamma\left(\frac{s+i t}{2}\right)$, so the analytic conductor of $L(s+i t, \chi)$, as defined in (6), is $C=\frac{q}{2 \pi} \sqrt{\frac{1}{4}+t^{2}}$. For any $t, \log C \leq \log q\left(\frac{1}{2}+|t|\right)$. Therefore, Corollary 1.1 can be applied to $\tilde{C}=q\left(\frac{1}{2}+|t|\right)$, and thus we obtain the corollary.

Proof of Corollary 1.3, $L(f, s)$ is an $L$-function of degree 2 with conductor $q$ and gamma factor

$$
\gamma(f, s)=\pi^{-s} \Gamma\left(\frac{s+(k-1) / 2}{2}\right) \Gamma\left(\frac{s+(k+1) / 2}{2}\right) .
$$

$L(f, s)$ satisfies Ramanujan's conjecture, and its analytic conductor $C$ is

$$
\frac{q}{\pi^{2}}\left(\frac{k}{2}-\frac{1}{4}\right)\left(\frac{k}{2}+\frac{3}{4}\right) \text {. }
$$

For $k \geq 1$, we have

$\log C=\log q k^{2}-\log 4 \pi^{2}+\log \left(1+\frac{1}{k}-\frac{3}{4 k^{2}}\right) \leq \log \tilde{C}-\log 4 \pi^{2}+\frac{1}{k}-\frac{3}{4 k^{2}} \leq \log \tilde{C}$.

Therefore, we can apply Corollary 1.1 to $\tilde{C}=q k^{2}$ and get the result.

Proof of Corollary 1.4. The Riemann zeta function $\zeta(s+i t)$ is an $L$-function of degree 1 . The gamma factor is $\gamma(s)=\pi^{-s / 2} \Gamma\left(\frac{s+i t}{2}\right)$. We can still apply Corollary 1.1. but we need to add a constant term derived from the pole $s=1-i t$. The analytic conductor $C$ is $\frac{1}{2 \pi} \sqrt{\frac{1}{4}+t^{2}}$. For $T \leq t \leq 2 T$ and $e^{20} \leq(\log \tilde{C})^{2} \leq T^{2}$,

$$
\log \tilde{C} \leq \log t+\log \frac{1}{2 \pi}+\frac{1}{8 t^{2}} \leq \log T+\log \frac{1}{\pi}+\frac{1}{8 t^{2}} \leq \log T .
$$

Therefore, we can apply Corollary 1.1 to $\tilde{C}=T$. Now we consider (7). For $\zeta(s+i t)$ it becomes

$$
\begin{aligned}
-\frac{\zeta^{\prime}}{\zeta}(s+i t)= & \frac{1}{2} \log \frac{q}{\pi^{d}}+\frac{1}{2} \sum_{j=1}^{d} \frac{\Gamma^{\prime}}{\Gamma}\left(\frac{s+k_{j}}{2}\right) \\
& -b-\sum_{\rho \neq 0,1}\left(\frac{1}{s-\rho}+\frac{1}{\rho}\right)+\frac{1}{s-1+i t}+\frac{1}{s+i t} .
\end{aligned}
$$

Following the proof of Theorem 2.1, we obtain that the contribution of the pole terms is bounded by $1.4 \cdot 10^{-6}$, which is negligible when we apply Corollary 1.1.

\section{Dedekind Zeta FunCtions}

Our upper bound can also be applied to Dedekind zeta functions. In this section, we will prove Corollary 1.5 .

Proof. The gamma factor of $\zeta_{K}(s)$ is

$$
\gamma(s)=\pi^{-d s / 2} \Gamma\left(\frac{s}{2}\right)^{r_{1}+r_{2}} \Gamma\left(\frac{s+1}{2}\right)^{r_{2}},
$$


where $r_{1}$ is the number of real embeddings of $K$ and $r_{2}$ is the number of pairs of complex embeddings, so that $d=r_{1}+2 r_{2}$.

Let $H(s)=\zeta_{K}(s)(s-1)$. Since the Dedekind zeta function has a simple pole at $s=1, H(s)$ is an entire function. Equation (8) for $H(s)$ is

$$
-\Re \frac{H^{\prime}}{H}(s)=\frac{1}{2} \log \frac{q}{\pi^{d}}+\frac{r_{1}+r_{2}}{2} \Re \frac{\Gamma^{\prime}}{\Gamma}\left(\frac{s}{2}\right)+\frac{r_{2}}{2} \Re \frac{\Gamma^{\prime}}{\Gamma}\left(\frac{s+1}{2}\right)+\Re \frac{1}{s}-G(s) .
$$

Similar to (10), we integrate the equality above as $\sigma$ varies from $\frac{1}{2}$ to $\sigma_{0}$, use Lemma 2.3, and obtain

$$
\log \left|H\left(\frac{1}{2}\right)\right|-\log \left|H\left(\sigma_{0}\right)\right| \leq \frac{\log C}{4 \log x}+\frac{d}{4 \log ^{2} x}-\frac{1}{2}\left(\sigma_{0}-\frac{1}{2}\right) G\left(\sigma_{0}\right)+\frac{1}{\log x} .
$$

Now we need an upper bound for $\log \left|H\left(\sigma_{0}\right)\right|$, which will be derived in the same way as the upper bound for $\log \left|L\left(f, \sigma_{0}\right)\right|$.

Lemma 3.1. Unconditionally, for any positive real number $t>\frac{1}{2}$ and any $x \geq 2$, we have

$$
\begin{aligned}
-\frac{H^{\prime}}{H}(t) \leq & \sum_{n \leq x} \frac{d \Lambda(n)}{n^{t}} \frac{\log \frac{x}{n}}{\log x}+\frac{1}{\log x}\left(\frac{H^{\prime}}{H}(t)\right)^{\prime}+\frac{1}{\log x} \sum_{\rho \neq 0,1} \frac{x^{\rho-t}}{(\rho-t)^{2}} \\
& +\frac{\left(r_{1}+r_{2}\right)}{\log x} \sum_{n=0}^{\infty} \frac{x^{-2 n-t}}{(2 n+t)^{2}}+\frac{r_{2}}{\log x} \sum_{n=0}^{\infty} \frac{x^{-2 n-1-t}}{(2 n+1+t)^{2}} .
\end{aligned}
$$

Proof. By the definition of $H(s)$ and by using the same arguments as in Lemma 2.4, we have

$$
\begin{aligned}
-\frac{H^{\prime}}{H}(t)= & -\frac{\zeta_{K}^{\prime}}{\zeta_{K}}(t)-\frac{1}{t-1} \\
\leq & \sum_{N \mathfrak{a} \leq x} \frac{\Lambda_{K}(\mathfrak{a})}{(N \mathfrak{a})^{t}} \frac{\log \frac{x}{N \mathfrak{a}}}{\log x}+\frac{1}{\log x}\left(\frac{H^{\prime}}{H}(t)\right)^{\prime} \\
& +\frac{1}{(t-1)^{2} \log x}+\frac{1}{\log x} \sum_{\rho \neq 0,1} \frac{x^{\rho-t}}{(\rho-t)^{2}} \\
& +\frac{\left(r_{1}+r_{2}\right)}{\log x} \sum_{n=0}^{\infty} \frac{x^{-2 n-t}}{(2 n+t)^{2}} \\
& +\frac{r_{2}}{\log x} \sum_{n=0}^{\infty} \frac{x^{-2 n-1-t}}{(2 n+1+t)^{2}}-\frac{x^{-t}}{t^{2} \log x}-\frac{x^{1-t}}{(t-1)^{2} \log x}-\frac{1}{t-1} .
\end{aligned}
$$

Furthermore,

$$
\sum_{N \mathfrak{a} \leq x} \frac{\Lambda_{K}(\mathfrak{a})}{(N \mathfrak{a})^{t}} \frac{\log \frac{x}{N \mathfrak{a}}}{\log x} \leq \sum_{n \leq x} \frac{d \Lambda(n)}{n^{t}} \frac{\log \frac{x}{n}}{\log x}
$$

and $\frac{1}{(s-1)^{2} \log x}-\frac{x^{1-s}}{(s-1)^{2} \log x}-\frac{1}{s-1}$ and $-\frac{x^{-s}}{s^{2} \log x}$ are holomorphic when $\mathbb{R} s>0$ and are less than or equal to zero for any positive real number $t$ and $x \geq 2$. Combining these facts and the inequalities above, we prove the lemma. 
We take $t=\sigma$ in Lemma 3.1 and integrate over $t$ from $\sigma_{0}$ to $\infty$. By the same reasoning as in the proof of Theorem 2.1.

$$
\begin{aligned}
& \log \left|H\left(\frac{1}{2}\right)\right| \leq \sum_{n \leq x} \frac{d \Lambda(n)}{n^{\frac{1}{2}+\frac{1}{2 \log x} \log n}} \frac{\log \frac{x}{n}}{\log x} \\
& +\frac{3 \log C}{4 \log x}+\frac{3 d}{4 \log ^{2} x}+\frac{4 d e^{-0.5}}{x^{1 / 2} \log ^{2} x}+\frac{2}{\log x+1}+\frac{1}{\log x} .
\end{aligned}
$$

From Corollary 1.1 (let $x=\log ^{2} C$ ), we obtain

$$
\log \left|\zeta_{K}\left(\frac{1}{2}\right)\right| \leq \frac{23 d}{25} \frac{\log C}{\log ^{2} \log C}+\frac{3}{8} \frac{\log C}{\log \log C}+\frac{3}{2 \log \log C}+\log 2 .
$$

The lemma follows because $\log \log C \geq 10$.

\section{Application to positive Definite ternary Quadratic Forms}

We call $N$ eligible for a positive ternary quadratic form $f(x, y, z)$ if there are no congruence obstructions prohibiting $f$ from representing $N$. Let $\chi=\left(\frac{-40 N}{\cdot}\right)$ be the Kronecker-Legendre symbol. Also define

$$
L(s, \chi):=\sum_{n=1}^{\infty} \frac{\chi(n)}{n^{s}} \quad \text { and } \quad L(E(-10 N), s):=\sum_{n=1}^{\infty} \frac{A(n) \chi(n)}{n^{s}} .
$$

By Dirichlet's class number formula (see [2]) and a special case of Waldspurger's theorem connecting the Fourier coefficients of half-integral weight cusp forms and $L(E(-10 N), 1)$ (Theorem 2 of [7]), Ono and Soundararajan showed that if $N$ is an eligible square-free integer coprime to 10 and is not represented by Ramanujan's form, then

$$
\frac{L(E(-10 N), 1)}{L(1, \chi)^{2}} \geq \frac{2}{7}\left(\frac{q}{4 \pi^{2}}\right)^{1 / 4},
$$

where $q$ is a conductor of $E(-10 N)$ and its value is $1600 N^{2}$ (see Prop. 2 in [7]). Ono and Soundararan proved that (17) failed when $N \geq 2 \cdot 10^{10}$. Note that for $N \leq 2 \cdot 10^{10}$, W. Galway verified by computer whether it is represented by the form. The difficulty of showing this is finding an upper bound for $L(E(-10 N), 1)$ because of the big contribution of zeros on the critical line. It involves long and complicated calculations.

As seen in the proof of Theorem 2.1, the technique from [1] allows us to ignore the contribution of nontrivial zeros. Therefore, by applying an upper bound in Theorem 2.1. not only is our calcuation much simpler but also we get a better lower bound for $N$, i.e. it requires $N \geq 3 \cdot 10^{7}$ to yield contradiction for (17).

With Ono and Soundararajan's methods of deriving (17) and the upper bound in Theorem 2.1. under the GRH, we may be able to obtain a not too large positive integer $N$ such that if $m \geq N$ and is represented by the spinor genus of a positive definite ternary form, then it is represented by the form itself. For example, in this paper, we will apply those techniques to Kaplansky's form and show that if $N \geq 2 \cdot 10^{8}$ is a square-free integer, then it is represented by Kaplansky's forms: $\varphi_{1}(x, y, z)=x^{2}+y^{2}+7 z^{2}$ and $\varphi_{2}(x, y, z)=x^{2}+2 y^{2}+2 y z+4 z^{2}$. This result will have a simpler proof and a better lower bound than the one that T. Reinke [8] derived. 
T. Reinke [8] applied the method in [7] to prove the analog of (17). If $N$ is an eligible square-free integer and is not represented by $\varphi_{j}$, where $j=1,2$, then

$$
\frac{L(E(-7 N), 1))}{L\left(1, \chi_{d}\right)^{2}} \geq \begin{cases}\frac{34}{101} \frac{q_{N}}{4 \pi^{2}} & \text { if }(N, 7)=1, \\ \frac{41}{101} \frac{q_{N}}{4 \pi^{2}} & \text { if }(N, 7)>1,\end{cases}
$$

where $q_{N}=28^{2} N^{2}$ for $(N, 7)=1$, and $q_{N}=7 \cdot\left(\frac{4}{7}\right)^{2} N^{2}$ for $(N, 7)>1$. The inequality (18) fails when $N \geq 10^{12}$.

Since the proof for both Ramanujan's form and Kaplansky's forms will be the same, for simplicity of notation let $L_{E}(s)$ be either $L(E(-10 N), s)$ or $L(E(-7 N), s)$, and let $L(s, \chi)$ be either $L\left(s, \chi_{-40 N}\right)$ or $L\left(s, \chi_{d}\right)$, where $d=-28 N$ when $(N, 7)=1$ and $d=-\frac{4 N}{7}$ when $(N, 7)>1$. We will get upper bounds for $L_{E}(1)$ and lower bounds for $L(1, \chi)$.

Let $L_{n}(s)=L_{E}\left(s+\frac{1}{2}\right)$. From the functional equation of $L_{E}(s)$, we have $\Lambda(s)=$ $\Lambda(1-s)$, where

$$
\Lambda(s)=q^{\frac{s}{2}} \pi^{-s} \Gamma\left(\frac{s+\frac{1}{2}}{2}\right) \Gamma\left(\frac{s+\frac{3}{2}}{2}\right) L_{n}(s) .
$$

Since $L_{E}(s)$ is an $L$-function of degree 2 and

$$
-\frac{L_{E}^{\prime}}{L_{E}}(s)=\sum_{n=1}^{\infty} \frac{\lambda(n) \chi(n)}{n^{s}}
$$

by Theorem 2.1 choosing $\lambda=0.5$, we obtain

$$
\begin{aligned}
\log \left|L_{E}(1)\right|= & \log \left|L_{n}\left(\frac{1}{2}\right)\right| \\
\leq & \Re \sum_{n \leq x} \frac{\lambda(n) \chi(n)}{n^{1+\frac{0.5}{\log x}} \log n} \frac{\log \left(\frac{x}{n}\right)}{\log x}+\frac{3}{4 \log x} \log \frac{q}{4 \pi^{2}}+\frac{3 \log 2}{4 \log x} \\
& +\frac{3}{2 \log ^{2} x}+\frac{8 e^{-0.5}}{x^{1 / 2} \log ^{2} x} .
\end{aligned}
$$

For a lower bound for $\log |L(1, \chi)|$, we prove the following proposition.

Proposition 4.1. Assume the GRH. Let $\chi$ be a primitive real character $\bmod q$, $y \geq 2, a(y)=\frac{1}{\log y}+\frac{2}{\sqrt{y} \log ^{2} y}$, and $b(y)=\frac{1}{1-\frac{2}{\sqrt{y} \log y}-\frac{2}{\log y}}$. We have

$$
\begin{aligned}
& \log |L(1, \chi)| \\
\geq & \sum_{n \leq y} \frac{\Lambda(n) \chi(n)}{n \log n} \frac{\log \left(\frac{y}{n}\right)}{\log y}+\frac{1}{4 \log y} \log \frac{q}{4 \pi^{2}}+\frac{\log 4}{4 \log y}-\frac{\gamma}{2 \log y} \\
& +a(y) b(y)\left(\sum_{n \leq y} \frac{\Lambda(n) \chi(n)}{n} \frac{\log \left(\frac{y}{n}\right)}{\log y}-\frac{1}{4} \log \frac{q}{4 \pi^{2}}-\frac{\pi^{2}}{24 \log y}-\frac{\log 4}{4}+\frac{\gamma}{2}\right) .
\end{aligned}
$$

Proof. Let $\rho=\frac{1}{2}+i \gamma$ run over the nontrivial zero of $L(s, \chi)$. Define

$$
F(s)=\Re \sum_{\rho} \frac{1}{s-\rho}=\sum_{\rho} \frac{\sigma-1 / 2}{(\sigma-1)^{2}+(t-\gamma)^{2}} .
$$

From (17) and (18) of Davenport [2], Chapter 12, if $L(s, \chi) \neq 0$,

$$
-\Re \frac{L^{\prime}}{L}(s, \chi)=\frac{1}{4} \log \frac{q}{\pi^{2}}+\frac{1}{2} \Re \frac{\Gamma^{\prime}}{\Gamma}\left(\frac{s+1}{2}\right)-F(s) .
$$


For $s \geq 1$, the contribution from the trivial zeros $\sum_{k=0}^{\infty} \frac{y^{-2 k-1-s}}{(2 k+1+s)^{2}} \geq 0$, and so by the same arguments as in Lemma (2.4), we have

$$
-\Re \frac{L^{\prime}}{L}(s, \chi) \geq \sum_{n \leq y} \frac{\Lambda(n) \chi(n)}{n^{s}} \frac{\log \left(\frac{y}{n}\right)}{\log y}+\frac{1}{\log y}\left(\frac{L^{\prime}}{L}(s, \chi)\right)^{\prime}+\frac{1}{\log y} \Re \sum_{\rho} \frac{y^{\rho-s}}{(\rho-s)^{2}} .
$$

Integrating (21) as $\sigma$ varies from 1 to infinity, using (20), and observing that

$$
\begin{aligned}
\Re \sum_{\rho} \int_{1}^{\infty} \frac{y^{\rho-\sigma}}{(\rho-\sigma)^{2}} d \sigma & \geq-\sum_{\rho} \int_{1}^{\infty} \frac{y^{1 / 2-\sigma}}{|\rho-1|^{2}} d \sigma \\
& =-\sum_{\rho} \frac{y^{-1 / 2}}{|\rho-1|^{2} \log y}=-\frac{2 y^{-1 / 2} F(1)}{\log y}
\end{aligned}
$$

we obtain

$\log |L(1, \chi)| \geq \sum_{n \leq y} \frac{\Lambda(n) \chi(n)}{n \log n} \frac{\log \left(\frac{y}{n}\right)}{\log y}+\frac{1}{4 \log y} \log \frac{q}{4 \pi^{2}}+\frac{\log 4}{4 \log y}-\frac{\gamma}{2 \log y}-a(y) F(1)$,

where $\frac{\Gamma^{\prime}}{\Gamma}(1)=-\gamma$.

To prove the proposition, we need to find the lower bound for $-F(1)$. First note that by taking the derivative of (20) at $s=1$, we get

$$
\Re\left(\frac{L^{\prime}}{L}(1, \chi)\right)^{\prime}=-\frac{1}{4}\left(\frac{\Gamma^{\prime}}{\Gamma}\right)^{\prime}(1)-\Re \sum_{\rho} \frac{1}{(1-\rho)^{2}} \geq-\frac{\pi^{2}}{24}-2 F(1) .
$$

From (20), (21) at $s=1$ and (23),

$$
\begin{aligned}
-F(1) \geq & \sum_{n \leq y} \frac{\Lambda(n) \chi(n)}{n} \frac{\log \left(\frac{y}{n}\right)}{\log y}-\frac{\pi^{2}}{24 \log y}-\frac{2 F(1)}{\log y} \\
& +\Re \frac{1}{\log y} \sum_{\rho} \frac{y^{\rho-1}}{(\rho-1)^{2}}-\frac{1}{4} \log \frac{q}{\pi^{2}}+\frac{\gamma}{2} \\
\geq & \sum_{n \leq y} \frac{\Lambda(n) \chi(n)}{n} \frac{\log \left(\frac{y}{n}\right)}{\log y}-\frac{\pi^{2}}{24 \log y} \\
& -\frac{2 F(1)}{\log y}-\frac{2 F(1)}{\sqrt{y} \log y}-\frac{1}{4} \log \frac{q}{4 \pi^{2}}-\frac{\log 4}{4}+\frac{\gamma}{2} .
\end{aligned}
$$

Therefore,

$$
-F(1) \geq b(y)\left(\sum_{n \leq y} \frac{\Lambda(n) \chi(n)}{n} \frac{\log \left(\frac{y}{n}\right)}{\log y}-\frac{\pi^{2}}{24 \log y}-\frac{1}{4} \log \frac{q}{4 \pi^{2}}-\frac{\log 4}{4}+\frac{\gamma}{2}\right) .
$$

Putting the inequality above into (22), we prove the proposition. 
To get an upper bound for $\log \frac{\left|L_{E}(1)\right|}{\left.L(1, \chi)\right|^{2}}$, we choose $x=600$ in (19) and $y=2100$ in Proposition 4.1 Thus,

$$
\begin{aligned}
\log \frac{\left|L_{E}(1)\right|}{|L(1)|^{2}} \leq & 0.147695+0.14158 \log \frac{q}{4 \pi^{2}} \\
& +\sum_{\substack{n=p^{k} \\
p \leq 600}}\left(\frac{\lambda(n)}{n^{0.5 / \log x \log n}} \frac{\log \left(\frac{x}{n}\right)}{\log x}-\frac{2 \Lambda(n)}{\log n} \frac{\log \left(\frac{y}{n}\right)}{\log y}\right. \\
& \left.-2 a(y) b(y) \Lambda(n) \frac{\log \left(\frac{y}{n}\right)}{\log y}\right) \frac{\chi(n)}{n} \\
& +\sum_{n=p=601}^{2100}\left|\frac{2}{\log n}+2 a(y) b(y)\right| \frac{\Lambda(n)}{n} \frac{\log \left(\frac{y}{n}\right)}{\log y} .
\end{aligned}
$$

To calculate the first sum, we use the exact value of $\lambda(n)$ of $L_{E}(s)$ (see [7, section 2, for Ramanujan's form and [8], section 3.2, for Kaplansky's form). Using a computer, for Ramanujan's form we get a contradiction for (17) when $N \geq 3 \cdot 10^{7}$. Similarly, for Kaplansky's forms, (18) fails when $N \geq 2 \cdot 10^{8}$.

\section{Appendix: Proof of lemma 2.3}

Proof. From [1, p. 202], we have

$$
\Re \frac{\Gamma^{\prime}}{\Gamma}(z)=\log |z|-\Re \frac{1}{2 z}+I(z),
$$

where

$$
I(z)=-\Re \int_{0}^{\infty} \frac{2 \eta}{\eta^{2}+z^{2}} \cdot \frac{d \eta}{e^{2 \pi \eta}-1}=-\int_{0}^{\infty} \frac{2 \eta\left(\eta^{2}+x^{2}-y^{2}\right)}{\left(\eta^{2}+x^{2}-y^{2}\right)^{2}+4 x^{2} y^{2}} \frac{d \eta}{e^{2 \pi \eta}-1} .
$$

If $y^{2}-x^{2} \leq 0$, it is clear that $I(z) \leq 0$, and $\Re \frac{1}{2 z} \geq 0$; hence the lemma is proved. Now we assume $y^{2}-x^{2}>0$. There are two possiblities for this case.

Case 1. $|z| \geq 4$.

To prove the lemma, first we will show that

$$
I(z) \leq \frac{15}{128|z|^{2}}+\frac{4 e^{-2 \pi|z| / 3}}{3}\left(1+\frac{1}{|z|^{2}}\right) .
$$

Integrating $I(z)$ by parts, we obtain

$$
\begin{aligned}
-\Re \int_{0}^{\infty} \frac{2 \eta}{\eta^{2}+z^{2}} \cdot \frac{d \eta}{e^{2 \pi \eta}-1} & =\frac{1}{\pi} \Re \int_{0}^{\infty} \frac{z^{2}-\eta^{2}}{\left(\eta^{2}+z^{2}\right)^{2}} \log \left(1-e^{-2 \pi \eta}\right) d \eta \\
& \leq \frac{1}{\pi} \int_{0}^{|z| / 3}+\int_{|z| / 3}^{\infty} \frac{\left|z^{2}-\eta^{2}\right|}{\left|\eta^{2}+z^{2}\right|^{2}} \log \left(\frac{1}{1-e^{-2 \pi \eta}}\right) d \eta .
\end{aligned}
$$

In the first integral $\left|z^{2}-\eta^{2}\right| \leq \frac{10}{9}|z|^{2},\left|\eta^{2}+z^{2}\right| \geq \frac{8}{9}|z|^{2}$, and $\int_{|a|}^{\infty} \log \left(\frac{1}{1-e^{-2 \pi \eta}}\right) d \eta \leq$ $\frac{\pi}{12 e^{2 \pi|a|}}$. Hence

$$
\frac{1}{\pi} \int_{0}^{|z| / 3} \frac{\left|z^{2}-\eta^{2}\right|}{\left|\eta^{2}+z^{2}\right|^{2}} \log \left(\frac{1}{1-e^{-2 \pi \eta}}\right) d \eta \leq \frac{15}{128|z|^{2}} .
$$


In the second integral $\frac{\left|z^{2}-\eta^{2}\right|}{\left|\eta^{2}+z^{2}\right|^{2}} \leq \frac{1}{\left|\eta^{2}+z^{2}\right|^{2}}+\frac{|z|^{2}}{\left|\eta^{2}+z^{2}\right|^{2}}$. Also $\left|\eta^{2}+z^{2}\right|=|z-i \eta| \cdot|z+i \eta| \geq$ $\frac{|z|}{4}$. Therefore

$$
\frac{1}{\pi} \int_{|z| / 3}^{\infty} \frac{\left|z^{2}-\eta^{2}\right|}{\left|\eta^{2}+z^{2}\right|^{2}} \log \left(\frac{1}{1-e^{-2 \pi \eta}}\right) d \eta \leq \frac{4 e^{-2 \pi|z| / 3}}{3}\left(1+\frac{1}{|z|^{2}}\right) .
$$

Combining the inequalities above, we proved (24). Since $x \geq 1 / 4$ and $|z| \geq 4$, by (24),

$$
-\Re \frac{1}{2 z}+I(z) \leq-\frac{1}{8|z|^{2}}+\frac{15}{128|z|^{2}}+\frac{4 e^{-2 \pi|z| / 3}}{3}\left(1+\frac{1}{|z|^{2}}\right) \leq 0 .
$$

Case 2. $|z|<4$.

From the last expression of (24), the integrand of $I(z)$ is greater than or equal to zero when $\eta \leq \sqrt{y^{2}-x^{2}}$. Hence

$$
-\Re \frac{1}{2 z}+I(z) \leq-\frac{x}{2\left(x^{2}+y^{2}\right)}-\int_{0}^{\sqrt{y^{2}-x^{2}}} \frac{2 \eta\left(\eta^{2}+x^{2}-y^{2}\right)}{\left(\eta^{2}+x^{2}-y^{2}\right)^{2}+4 x^{2} y^{2}} \frac{d \eta}{e^{2 \pi \eta}-1} .
$$

Let $f(x, y)$ be the right-hand side of the inequality above. Once we show that $f(x, y) \leq 0$, the lemma will be proved. Without loss of generality, we can assume that $y \geq 0$. For any fixed $y>x$,

$$
\frac{\partial}{\partial x} f(x, y)=\frac{2 x^{2}-2 y^{2}}{4\left(x^{2}+y^{2}\right)^{2}}+\int_{0}^{\sqrt{y^{2}-x^{2}}} \frac{2 \eta}{e^{2 \pi \eta}-1} \frac{2 x\left(\eta^{2}+x^{2}-y^{2}\right)^{2}+8 x y^{2}\left(\eta^{2}-y^{2}\right)}{\left(\left(\eta^{2}+x^{2}-y^{2}\right)^{2}+4 x^{2} y^{2}\right)^{2}} d \eta .
$$

Since $y^{2}-x^{2}>0$, the first term is less than 0. Also $2 x\left(\eta^{2}+x^{2}-y^{2}\right)^{2}+8 x y^{2}\left(\eta^{2}-y^{2}\right)<$ 0 because $2 y \sqrt{y^{2}-\eta^{2}}>y^{2}-\eta^{2} \geq y^{2}-x^{2}-\eta^{2}$. Therefore, $f(x, y)$ is decreasing with respect to $x$. Because $x \geq 1 / 4$, for any fixed $y>x, f(x, y) \leq f(1 / 4, y)$. We know that $|z|<4$. From numerical computation, $f(1 / 4, y) \leq 0$ for $1 / 4 \leq y \leq 4$.

\section{ACKNOWLEDGEMENTS}

The author is very grateful to Professor Soundararajan for his guidance throughout the writing of this paper. He would like to thank Xiannan Li for helpful editorial comments. He also wants to thank the referee for a careful reading of this paper and helpful comments.

\section{REFERENCES}

[1] L. Ahlfors, Complex Analysis, McGraw-Hill, New York, 1978. MR510197 (80c:30001)

[2] H. Davenport, Multiplicative Number Theory, Graduate Texts in Mathematics, vol. 74, Springer-Verlag, New York, 2000. MR,1790423 (2001f:11001)

[3] W. Duke and R. Schulze-Pillot, Representation of integers by positive ternary quadratic forms and equidistribution of lattice points on ellipsoids, Invent. Math. 99, 1, 49-57, 1990. MR1029390 (90m:11051)

[4] G. Harcos, Uniform approximate functional equation for principal L-functions, Internat. Math. Res. Notices 18, 923-932, 2002. MR1902296 (2003d:11074)

[5] H. Iwaniec and E. Kowalski, Analytic Number Theory, American Mathematical Society Colloquium Publications, vol. 53, Amer. Math. Soc., Providence, RI, 2004. MR2061214 (2005h:11005)

[6] J. Kelley, Kaplansky's ternary quadratic form, Int. J. Math. Math. Sci. 25, 289-292, 2001. MR.1812392(2002c:11038)

[7] K. Ono and K. Soundararajan, Ramanujan's ternary quadratic form. Invent. Math. 130, no. 3, 415-454, 1997. MR 1483991 (99b:11036) 
[8] T. Reinke, Darstellbarkeit ganzer Zahlen durch Kaplanskys ternäre quadratische Form, Ph.D. thesis, Fachbereich Mathematik und Informatik, Universität Münster, 2003.

[9] J. Barkley Rosser and L. Schoenfeld, Sharper bounds for the Chebyshev functions $\theta(x)$ and $\psi(x)$, Collection of articles dedicated to Derrick Henry Lehmer on the occasion of his seventieth birthday. Math. Comp. 29, 243-269, 1975. MR.0457373(56:15581a)

[10] G. Shimura, On modular forms of half integral weight. Ann. of Math. (2) 97, 440-481, 1973. MR0332663 (48:10989)

[11] K. Soundararajan, Moments of the Riemann zeta-function, Ann. of Math. (2) 170, 2009.

[12] H. M. Stark, The analytic theory of algebraic numbers. Bull. Amer. Math. Soc. 81, no. 6, 961-972, 1975. MR0444611 (56:2961)

[13] E. C. Titchmarsh, The Theory of the Riemann Zeta-function, Oxford University Press, New York, 1986. MR882550 (88c:11049)

Department of Mathematics, Stanford University, 450 Serra Mall, Building 380, Stanford, CaLifornia 94305

E-mail address: vchandee@math.stanford.edu 